\title{
LINFADENECTOMÍA RETROPERITONEAL LAPAROSCÓPICA EN CÁNCER DE TESTÍCULO NO SEMINOMA ESTADIO I.
}

\author{
Octavio A. Castillo', Juan Manuel Álvarez, Gonzalo Vitagliano, David Ramírez, Manuel Díaz y \\ Rafael Sánchez-Salas.
}

Unidad de Endourología y Laparoscopía Urológica. Clínica Santa María.

Departamento de Urología'. Facultad de Medicina. Universidad de Chile. Chile.

\begin{abstract}
Resumen.- OBJETIVOS: Se presenta una serie de pacientes con cáncer de testículo no seminoma estadio I sometidos a linfadenectomía retroperitoneal por vía laparoscópica.

MÉTODOS: Entre marzo de 1993 y enero de 2004, 111 pacientes con diagnóstico de cáncer de testículo no seminomatoso estadio / fueron intervenidos quirúrgicamente por linfadenectomía retroperitoneal laparoscópica (LRP-L). Todos los procedimientos fueron realizados por el mismo cirujano (O.A.C). Los datos clínicos fueron recolectados de forma prospectiva en una base de datos creada para tal fin. Se analizaron la características del grupo de pacientes, tiempo operatorio, sangrado, tiempo de hospitalización, complicaciones operatorias y perioperatorias, tasa de conversión a cirugía abierta y seguimiento.
\end{abstract}

RESULTADOS: Se realizaron 111 LRP-L en 111 pacientes con edad promedio de 29.3 años (rango 1544 años). 61 procedimientos correspondieron a LRP-L derecha y 50 a LRP-L izquierda. El tiempo operatorio promedio fue de 138 minutos (rango 60-300 minutos). Se presentaron complicaciones intraoperatorias en 10 pacientes $(9 \%)$, de las cuales, 9 correspondieron a lesiones vasculares $(8.1 \%)$ y 1 a lesión duodenal $(0.9 \%)$. Se realizo conversión a cirugía abierta en 3 pacientes (2.7\%). El tiempo promedio de hospitalización fue de 43. 3 horas (rango 24-120). 5 pacientes (4.5\%) presentaron recurrencia del tumor, con un seguimiento promedio de 30 meses (rango 24-94 meses). Las recurrencias se ubicaron a nivel retroperitoneal, en mediastino y a nivel pulmonar. El tiempo de recurrencia oscilo entre 4 y 64 meses.

CONCLUSIONES: La LRP-L ofrece resultados oncológicos equivalentes a los obtenidos con cirugía abierta, con la ventaja de ser mínimamente invasiva y consecuentemente disminuir la morbilidad. La linfadenectomía retroperitoneal laparoscópica es una buena alternativa para el manejo de los pacientes con cáncer de testículo en estadio 1.

Palabras clave: Tumor testicular. Laparoscopia. Linfadenectomía.

Summary.- OBJECTIVES: We report a series of patients with stage I non seminomatous testicular cancer in whom laparoscopic retroperitoneal lymphadenectomy was carried out.

METHODS: Between March 1993 and January 2004 100 and patients with the diagnosis of stage I non seminomatous testicular cancer underwent laparoscopic retroperitoneal lymphadenectomy (LRPL). All operations were performed by the same surgeon (O.A.C). Clinical 
data were collected prospectively in a database. Patients' demographic data, operative time, estimated blood loss, hospital stay, operative and post operative complications, convention rate and follow-up were all analyzed.

RESULTS: 111 LRPL were performed in 111 patients with a mean age of 29.3 years (range 15-44 years). 61 procedures were right-LRPL and 50 left-LRPL. Mean operative time was 138 minutes (range 60-300 minutes). Intraoperative complications appeared in 10 cases (9\%), 9 vascular complications (8. $1 \%$ ) and 1 duodenal lesion $(0.9 \%)$. Conversion to open surgery was necessary in three patients $(2.7 \%)$. Mean hospital stay was 43.3 hours (range 24-120). 5 patients (4.5\%) had tumor recurrence, with a mean follow-up of 30 months (range 24-94 months). Recurrences were localized in the retroperitoneum, mediastinum and lungs. Recurrence time varied between four and 64 months.

CONCLUSIONS: LRPL offers oncological results equivalent to open surgery, with the advantage of being minimally invasive and its consequent decrease in morbidity. The laparoscopic retroperitoneal lymphadenectomy is a good option for the management of patients with stage 1 testicular cancer.

Keywords: Testicular tumor. Laparoscopy. Lymphadenectomy.

\section{INTRODUCCIÓN}

El retroperitoneo es el sitio inicial de metástasis en los pacientes con diagnóstico de tumor testicular de células germinales no seminomatoso (TGNS). Un 25 a $30 \%$ de los pacientes en estadío clínico I presentarán compromiso ganglionar retroperitoneal (1). Existe controversia sobre la estrategia terapéutica a seguir tras la realización de orquiectomia radical. Las alternativas disponibles en la actualidad incluyen la aplicación de esquemas de vigilancia, quimioterapia primaria y la linfadenectomía retroperitoneal (LRP) (2). La LRP es el único método que permite realizar de forma objetiva la estadificación tumoral y por lo tanto permite la indicación del tratamiento mas adecuado en función del estadío. Casi dos tercios de los pacientes afectados por este tipo de neoplasia, no ameritarían la aplicación de otro tipo de tratamientos luego del hallazgo de ganglios positivos tras la realización de LRP. El principal inconveniente que presenta la LRP es su morbilidad, más aun si se considera que en un significativo porcentaje de los pacientes constituye un procedimiento meramente diagnóstico. Tanto la vigilancia como la quimioterapia primaria como opciones terapéuticas, tienen como propósito evitar los riesgos y posibles complicaciones asociadas a la LRP (2). El advenimiento de la LRP-L, en los últimos años, ofrece las ventajas de la disección ganglionar con reducción de la morbilidad, por lo tanto, constituye la alternativa quirúrgica con índices de eficacia y costos comparables al procedimiento convencional $(1,3,4)$. Se presenta una serie institucional de 111 pacientes con diagnóstico de TGNS en estadío I, que fueron tratados mediante LRP-L transperitoneal.

\section{MATERIAL Y MÉTODOS}

Entre marzo de 1993 y enero de 2004 se realizaron 111 LRP-L transperitoneales en 111 pacientes con diagnostico de TGNS en estadío I. Todos los procedimientos fueron realizados o supervisados por el mismo cirujano (O.A.C.). En nuestra institución, se han realizado hasta la fecha un total de 232 LRP-L. La presente serie comprende de forma exclusiva los pacientes tratados entre 1993 y 2004, con la finalidad de ofrecer un periodo de seguimiento mínimo de 24 meses.

\section{Técnica quirúrgica:}

\section{Preparación preoperatoria}

A todos los pacientes se indica dieta hídrica el día previo a la cirugía, así como la colocación de enema rectal unas horas antes del procedimiento. Una vez inducida la anestesia general, se colocan una sonda uretrovesical y sonda nasogástrica.

\section{Posición del paciente}

El paciente es ubicado en decúbito lateral izquierdo o derecho según el lado en que esté indicada la disección retroperitoneal y de la misma forma se fija a la mesa operatoria, con el respectivo soporte en los miembros superiores e inferiores. El posicionamiento adecuado facilita el acceso al retroperitoneo debido al desplazamiento del intestino hacia la línea media.

\section{Procedimiento operatorio}

La creación del neumoperitoneo se realiza por punción a nivel subcostal mediante técnica de Veress. La ubicación de los puertos de trabajo presenta variaciones según el procedimiento se realice del lado derecho o izquierdo.

\section{LRP-L derecha:}

En este lado el puerto de la óptica se ubica en posición pararectal y paraumbilical derecha. Se 


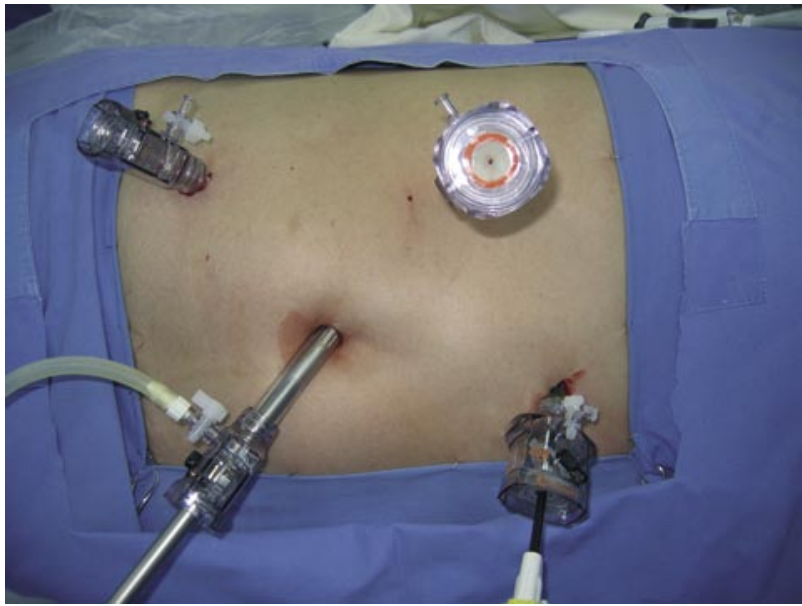

FIGURA 1. Posicionamiento de trócares para la linfadenectomía retroperitoneal derecha.

utiliza óptica de $30^{\circ}$ que ofrece un campo visual idóneo. Los trocares adicionales son un trocar de $10 \mathrm{~mm}$ subcostal y un trocar de $5 \mathrm{~mm}$ en fosa ilíaca derecha, ambos a nivel de la línea medio-clavicular. Un trocar adicional de $5 \mathrm{~mm}$ se coloca en posición epigástrica para separación hepática. Eventualmente, este trocar se puede utilizar para separar el colon en la disección más distal. (Figura 1).

\section{LRP-L izquierda:}

En el lado izquierdo el puerto para la óptica se ubica en posición pararectal y paraumbilical izquierda. Los puertos de trabajo corresponden a un trocar de $10 \mathrm{~mm}$ en fosa ilíaca izquierda y uno de 5 $\mathrm{mm}$ subcostal en la línea medio-clavicular. Un trocar adicional de $5 \mathrm{~mm}$ para separación de estructura, se coloca en el flanco, completándose así un romboide $(5,6)$. Este trocar adicional es colocado una vez lograda una amplia movilización del colon ascendente o descendente según el caso. (Figura 2).

Una vez colocados los puertos de trabajo, se procede a la liberación del colon ascendente o descendente, incidiendo la línea de Toldt. Una vez lograda una amplia movilización del colon, se procede a la identificación de las referencias anatómicas claves como son: uréteres, vena cava, aorta y vasos renales. No utilizamos de rutina cateterización ureteral previa, y no la consideramos necesaria. Inicialmente, luego de identificar las referencias anatómicas, se procede a la disección y resección de los vasos gonadales. La extensión de la disección linfática es la misma descrita para las disecciones realizadas a cielo abierto, siguiendo las plantillas y los límites de disección descritos por Weissbach y Boedefeld (7) (Figura 3) con la salvedad, que desde la demostra-

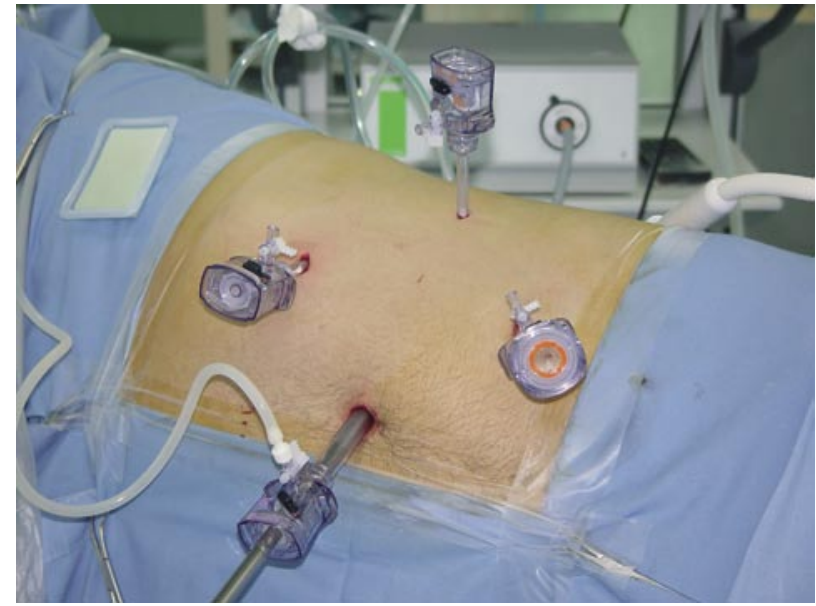

FIGURA 2. Posicionamiento de trócares para la linfadenectomía retroperitoneal izquierda.

ción por Holtl y cols. (8) que la diseminación linfática primaria se desarrolla siempre ventral a los vasos lumbares, no realizamos la sección de los mismos para lograr la remoción del tejido linfático por detrás de aorta y vena cava. Esta circunstancia permite reducir el tiempo operatorio y el riesgo de lesiones vasculares de difícil manejo. Para tumores del lado derecho, se incluye el tejido linfático paracavo, precavo e interaortocavo, desde la arteria renal derecha por arriba y hasta la bifurcación ilíaca por debajo (Figura 4). Para tumores izquierdos, la disección comprende todo el tejido linfático paraaórtico desde los vasos renales por arriba hasta la arteria ilíaca

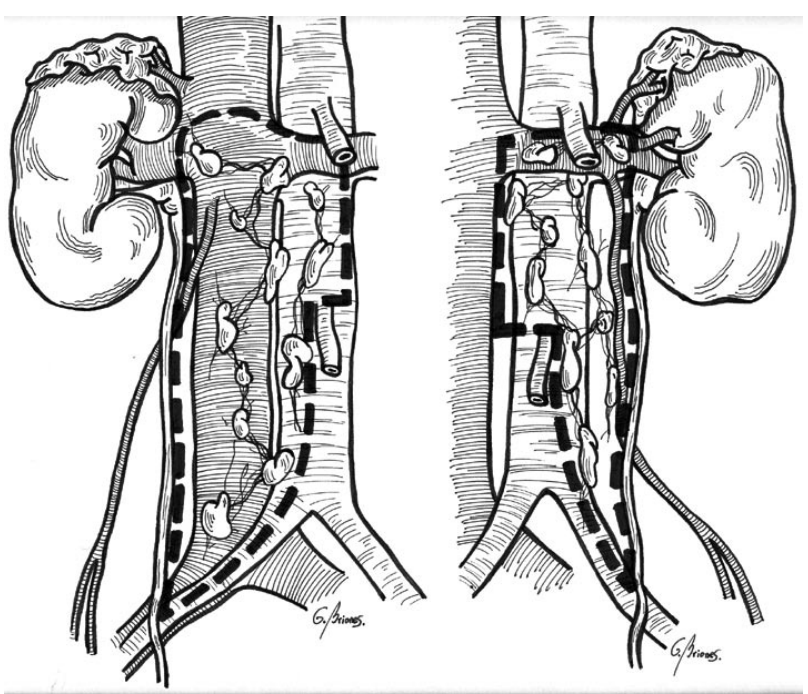

FIGURA 3. Plantillas y límites de disección descritos por Weissbach y Boedefeld. 


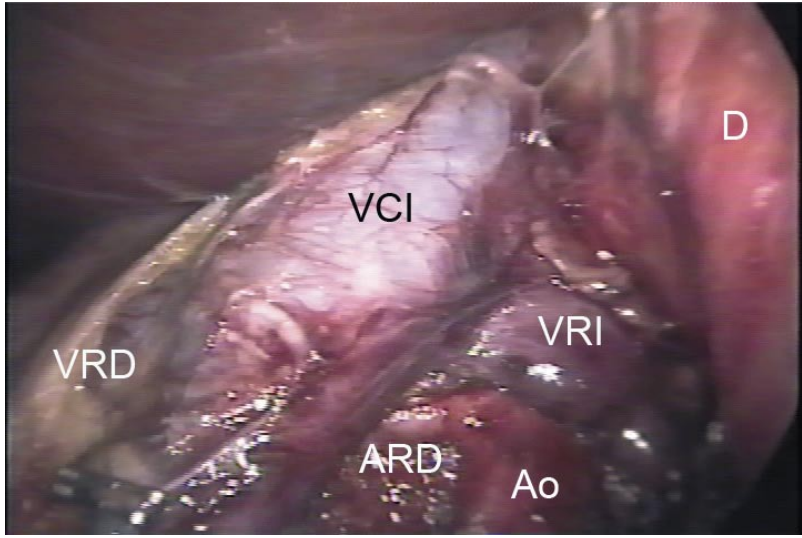

FIGURA 4. Linfadenectomía retroperitoneal derecha, visión endoscópica. VRD- Vena renal derecha, VClVena cava inferior, ARD-Arteria renal derecha, VRIVena renal izquierda, Ao- Arteria aorta, D-Duodeno.

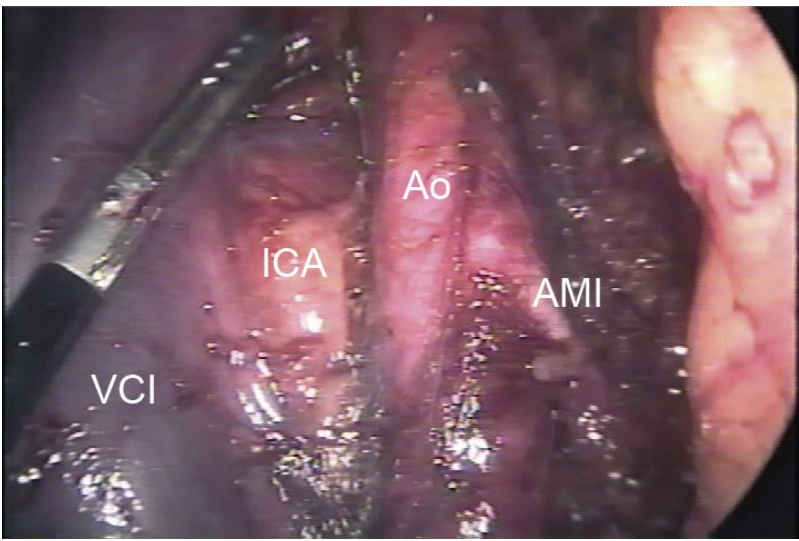

FIGURA 5. Linfadenectomía retroperitoneal izquierda, visión endoscópica. VCl- Vena cava inferior, ICA-Espacio interaortocavo, Ao- Arteria Aorta, AMl- Arteria mesentérica inferior. izquierda por abajo, incluyendo el tejido preaórtico en la zona de la arteria mesentérica inferior (Figura 5). En general, preferimos iniciar la extirpación del tejido linfático desde los vasos ilíacos, para avanzar en sentido cefálico, lo que en nuestra experiencia facilita la cirugía. La utilización del bisturí ultrasónico (Ethicon-EndosurgeryTM) permite ejecutar una prolija coagulación y disección del retroperitoneo. Siempre se realiza una técnica de preservación de los nervios simpáticos. Una vez finalizada la disección, el espécimen es retirado de la cavidad abdominal en una bolsa de polietileno. De rutina se sutura la aponeurosis de todos los portales de trabajo. Las sondas uretrovesical y nasogástrica son retiradas al término de la cirugía, y el paciente reinicia la vía oral a las 6 horas de finalizado el procedimiento.

\section{RESULTADOS}

Se realizaron un total de 111 LRP-L en 111 pacientes con TGNS en estadio clínico I. La edad promedio fue de 29.3 años con un rango de 15 a 44 años. La linfadenectomía fue derecha en 61 casos e izquierda en 50. Los resultados de la serie se resumen en la Tabla I. El tiempo operatorio fue en promedio de 138 minutos, con un rango de 60 a 300 minutos. Hubo un total de 10 complicaciones intraoperatorias en 10 pacientes $(9.0 \%)$, siendo la mayoría de estas complicaciones de tipo vascular (Tabla II). En 3 de estos pacientes fue necesaria una conversión a cirugía abierta $(2.7 \%)$. Las causas que indicaron la conversión fueron, desgarro de vena lumbar al término de la linfadenectomía, lesión de cava en la colocación

TABLA I. RESULTADOS.

\begin{tabular}{|l|l|}
\hline Tiempo operatorio $(\mathrm{min})$ & $138.9(60-300)$ \\
\hline Sangrado $(\mathrm{ml})$ & $110.9(0-3000)$ \\
\hline Conversión (nro. pacientes) & $3(2.7 \%)$ \\
\hline Tiempo de hospitalización (horas) & $43.3(24-120)$ \\
\hline Número de ganglios obtenidos $(\mathrm{n})$ & $13.7(4-36)$ \\
\hline Pacientes con ganglios positivos $(\mathrm{n})$ & $18(16.2 \%)$ \\
\hline Recurrencia & 5 pacientes $(4.5 \%)$ \\
\hline Tiempo de seguimiento (meses) & $30(1-94)$ \\
\hline
\end{tabular}


TABLA II. COMPLICACIONES INTRAOPERATORIAS.

\begin{tabular}{|ll|}
\hline Complicación & Número ( \%) \\
\hline Lesión vascular & $9(8.1 \%)^{*}$ \\
\hline Lesión duodenal. & $1(0.9 \%)$ \\
\hline Total & $10(9.0 \%)$ \\
\hline
\end{tabular}

*tres pacientes requirieron conversión a cirugía abierta

del primer trocar y una lesión por quemadura del gancho monopolar de la arteria iliaca. Esta última lesión requirió de un injerto de vena safena para su corrección. El sangrado promedio de la serie fue de $110.9 \mathrm{ml}$ con un rango de 0 a $3000 \mathrm{ml}$, requiriendo transfusión en 2 casos (1.8\%). El número promedio de ganglios linfáticos obtenidos fue de 13.7, con un rango de 4 a 36 ganglios. Fueron identificadas metástasis linfáticas en 18 pacientes (16.2\%), 12 de los cuales tenían factores de riesgo en la biopsia original (predominancia de carcinoma embrionario y/o invasión vascular o linfática). Todos los pacientes con metástasis ganglionares fueron tratados en forma complementaria con quimioterapia con dos ciclos de bleomicina, etoposido y cisplatino. Dos pacientes desarrollaron un linfocele durante el postoperatorio y ambos casos fueron manejados en forma conservadora. La vía oral fue iniciada en todos los casos a las 6 horas de la cirugía. Todos los pacientes fueron manejados con analgésicos no esteroideos. El tiempo de hospitalización fue en promedio de 43.36 horas, con un rango de 24 a 120 horas. No hubo mortalidad en la serie. El tiempo de seguimiento promedio de la serie es de 30.07 meses, con un rango de 24 a 94 meses. En el grupo de pacientes con estadío patológicol, hemos tenido 5 recurrencias de la enfermedad $(4.5 \%)$, de las cuales 3 ocurrieron en el retroperitoneo exclusivamente, 1 en retroperitoneo y mediastino de forma simultánea y 1 en parénquima pulmonar solamente. El tiempo de recurrencia varió entre 4 y 64 meses. Todos los pacientes han conservado la eyaculación.

\section{DISCUSIÓN}

De las opciones terapéuticas planteadas para el manejo de los pacientes con TGNS en estadío I, los esquemas de vigilancia mediante la realización periódica de examen físico, radiografías de tórax, medición de marcadores tumorales y tomografía computada (TC) de abdomen y pelvis, cuentan con la ventaja de prevenir morbilidad innecesaria en un porcentaje importante de pacientes (70-75\%). Estos podrían nunca desarrollar enfermedad en el retroperitoneo. Por otro lado, la aparición de metástasis durante el período de vigilancia permite la aplicación de esquemas de quimioterapia con altas tasas de curación cercanas al 99\% (9-11). La principal desventaja de esta opción, radica en el incumplimiento de los esquemas de seguimiento y la eventual pérdida de la posibilidad de un tratamiento curativo en aquellos pacientes que desarrollen metástasis (9).

Existen esquemas de quimioterapia adaptada al riesgo para el tratamiento de pacientes con TGNS en estadío I con tasas de curación similares a las demás alternativas $(9,11)$. El principal inconveniente de esta modalidad terapéutica es que aún no se han logrado definir con certeza cuales son los factores de riesgo que permiten predecir que pacientes desarrollaran metástasis $(9,11)$. Con los esquemas actuales un porcentaje cercano al $50 \%$ de los pacientes recibirá quimioterapia innecesaria con un número no despreciable de eventos adversos y toxicidad a largo plazo $(9,11)$. Es por esto, que muchos autores sostienen que ante la presentación de un paciente con TGNS en estadío I la pauta terapéutica más firme debe estar relacionada con la realización de una LRP - la aplicación de un esquema de vigilancia $(2,11)$. Dos aspectos se han asociado históricamente a la morbilidad del procedimiento de LRP, la morbilidad propia de cualquier laparotomía y las alteraciones en la eyaculación.

El procedimiento de LRP tradicional ha demostrado ser eficaz desde el punto de vista terapéutico con una tasa de complicaciones del $12 \%$ y una morbilidad a corto y largo plazo de 15\% en centros de experiencia en el manejo de este tipo de pacientes $(12,13)$. La morbilidad asociada a cualquier laparotomía ha logrado reducirse desde la introducción de la técnica laparoscópica para LRP (1). En nuestra experiencia, ha sido notable observar ausencia de íleo postoperatorio, lo que permite un inicio precoz de la vía oral y facilidad para el manejo del dolor postoperatorio, cuando se compara con la cirugía clásica a cielo abierto. En nuestra serie todos los pacientes pudieron ser manejados con analgésicos no esteroideos.

Los trastornos eyaculatorios asociados al procedimiento quirúrgico para el manejo de pacientes con TGNS en estadío I se han podido solucionar a través de la aplicación de la disección unilateral y mediante la utilización de técnicas de conservación nerviosa (14-16). En nuestra serie hemos podido conservar la eyaculación anterógrada en el 100\% de los pacientes sometidos a LRP-L. 


\begin{tabular}{|c|c|c|c|c|c|c|c|c|c|c|c|c|}
\hline 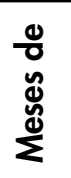 & 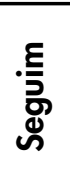 & $\begin{array}{l}\overline{\mathrm{g}} \\
\text { g } \\
\text { o }\end{array}$ & ڤે & $\begin{array}{l}\stackrel{\infty}{\infty} \\
\stackrel{1}{1} \\
\stackrel{\sim}{\simeq}\end{array}$ & \multicolumn{2}{|c|}{$\stackrel{\circ}{\forall}$} & $\stackrel{N}{N}$ & $\begin{array}{c}\underset{\text { N }}{\infty} \\
\stackrel{1}{1} \\
\stackrel{\infty}{ \pm}\end{array}$ & \multicolumn{2}{|c|}{$\underset{n}{\underline{\underline{z}}}$} & 이 & $\begin{array}{l}\nexists \\
\sigma \\
1 \\
=\end{array}$ \\
\hline 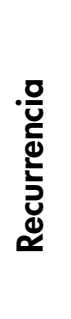 & $\stackrel{\circ}{=}$ & 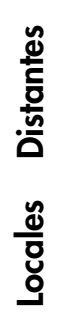 & $m$ & 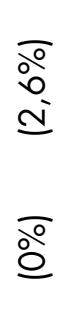 & 0 & $\begin{array}{l}\overline{0} \\
\infty \\
0 \\
0\end{array}$ & - & 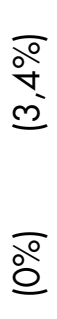 & 0 & $\begin{array}{l}\text { @ } \\
\text { ○ } \\
\stackrel{0}{0}\end{array}$ & . & $\begin{array}{l}\frac{*}{\circ 0} \\
\text { o } \\
= \\
\overline{0} \\
0 \\
0 \\
0\end{array}$ \\
\hline 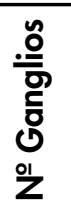 & 总 & $\stackrel{20}{\varrho}$ & 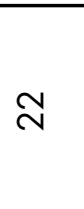 & $\begin{array}{l}\text { 吕 } \\
\stackrel{2}{2} \\
\stackrel{0}{=}\end{array}$ & \multicolumn{2}{|c|}{$\stackrel{\stackrel{\alpha}{z}}{z}$} & $\simeq$ & $\begin{array}{l}\bar{D} \\
\pm \\
\pm\end{array}$ & 으 & $\begin{array}{l}\overline{0} \\
\text { ○े } \\
\end{array}$ & $\stackrel{\infty}{-}$ & $\begin{array}{l}\overline{0} \\
\stackrel{\text { }}{0} \\
\stackrel{0}{=}\end{array}$ \\
\hline 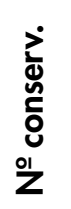 & 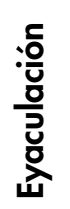 & $\stackrel{0}{0}$ & 으 & $\begin{array}{l}\bar{o} \\
\stackrel{\circ}{+} \\
\infty \\
\text { a }\end{array}$ & m & $\begin{array}{l}\overline{0} \\
\hat{\alpha} \\
\underline{\alpha}\end{array}$ & $\stackrel{\infty}{\sim}$ & $\begin{array}{l}\overline{0} \\
\text { in } \\
\stackrel{0}{0} \\
2\end{array}$ & ○ & $\begin{array}{l}\bar{\circ} \\
\stackrel{\circ}{\circ} \\
=\end{array}$ & 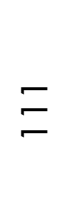 & $\begin{array}{l}\bar{\circ} \\
\text { ᄋ̆ } \\
\text { 을 }\end{array}$ \\
\hline 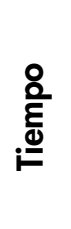 & $\begin{array}{l}\overline{\text { on }} \\
\text { 흠 } \\
\text { 우 }\end{array}$ & $\begin{array}{l}\text { on } \\
\text { 후 } \\
\text { 응 } \\
\text { o } \\
\text { 으 }\end{array}$ & \multicolumn{2}{|c|}{ 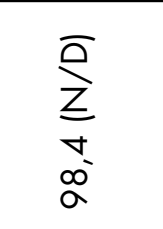 } & $\stackrel{N}{N}$ & 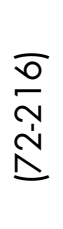 & \begin{tabular}{l}
\multirow{N}{*}{} \\
\end{tabular} & 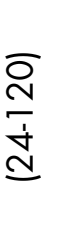 & & 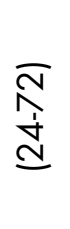 & $\stackrel{m}{\stackrel{P}{q}}$ & 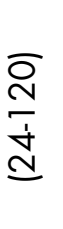 \\
\hline 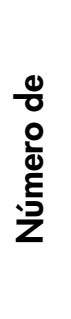 & 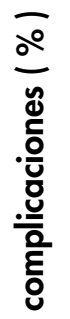 & 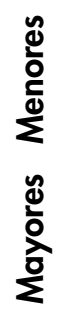 & $\stackrel{\circ}{\sim}$ & 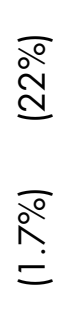 & & & $\sim$ & $\begin{array}{l}\overline{0} \\
\infty \\
0 \\
0 \\
0 \\
\infty \\
0 \\
0\end{array}$ & 0 & ㅇํㅇ & . & $\begin{array}{l}\bar{\circ} \\
\stackrel{\text { N }}{ } \\
\bar{\circ} \\
\stackrel{0}{0} \\
\stackrel{0}{0}\end{array}$ \\
\hline 总 & :0 & $\stackrel{0}{\circ}$ & $n$ & $\begin{array}{l}\overline{0} \\
0 \\
\stackrel{0}{\simeq}\end{array}$ & \multicolumn{2}{|c|}{$\stackrel{\stackrel{\alpha}{\Sigma}}{\text { z }}$} & $\sim$ & $\begin{array}{l}\overline{0} \\
\infty \\
0 \\
0\end{array}$ & 0 & $\begin{array}{l}\bar{\circ} \\
\stackrel{0}{0}\end{array}$ & $m$ & 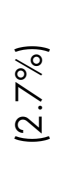 \\
\hline 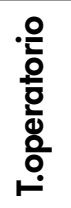 & 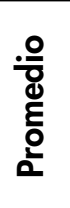 & 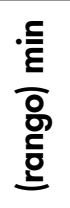 & $\stackrel{\circ}{\sim}$ & $\begin{array}{l}\bar{D} \\
\hat{1} \\
\text { م่ } \\
\text { 三 } \\
=\end{array}$ & \multicolumn{2}{|c|}{$\stackrel{\stackrel{\alpha}{z}}{Z}$} & $\stackrel{\infty}{\stackrel{\infty}{N}}$ & $\begin{array}{l}\overline{0} \\
\infty \\
\\
\\
\\
=\end{array}$ & $\stackrel{\text { }}{\text { ㄱ }}$ & $\begin{array}{l}\overline{8} \\
\text { ల్ } \\
\text { ᄋ } \\
\infty \\
=\end{array}$ & $\stackrel{\infty}{\stackrel{\infty}{ }}$ & $\begin{array}{l}\overline{8} \\
\text { ले } \\
\text { ô } \\
\underline{0}\end{array}$ \\
\hline 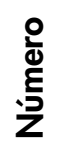 & $\stackrel{0}{0}$ & 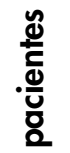 & $\stackrel{m}{=}$ & & \multicolumn{2}{|c|}{ স } & \multicolumn{2}{|c|}{ 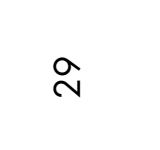 } & & ৩ & \multicolumn{2}{|c|}{$\Xi$} \\
\hline$\frac{\grave{0}}{\frac{1}{4}}$ & & & 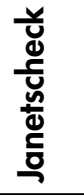 & $\begin{array}{l}\bar{D} \\
\dot{\dot{d}} \\
\dot{0} \\
\dot{\lambda}\end{array}$ & $\frac{\text { d }}{\overline{\bar{\sigma}}}$ & 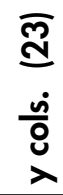 & 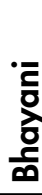 & 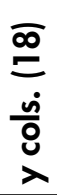 & $\begin{array}{l}\breve{g} \\
\text { o } \\
\text { s }\end{array}$ & $\begin{array}{l}\stackrel{\sigma}{=} \\
\dot{\dot{0}} \\
\dot{\delta} \\
\lambda\end{array}$ & 을 & $\begin{array}{l}\dot{0} \\
\mathbf{8}\end{array}$ \\
\hline
\end{tabular}

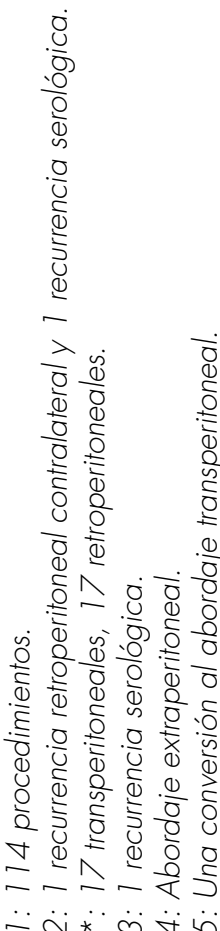


De manera similar a lo reportado por otros autores, las complicaciones más importantes que hemos observado en nuestra casuística estuvieron asociadas a la producción de lesiones vasculares, obligando a la conversión en tres oportunidades (17-19). Las seis lesiones vasculares restantes se pudieron controlar manteniendo el abordaje laparoscópico. Es importante mencionar que nuestra institución funciona como centro de entrenamiento de fellows y residentes. La gran mayoría de las lesiones vasculares descritas ocurrieron durante la curva de aprendizaje de estos.

En cuanto a los resultados oncológicos de nuestra serie hemos observado compromiso retroperitoneal (estadío patológico II), en 18 pacientes (16.2\%). En todos estos casos indicamos tratamiento adyuvante con dos ciclos de bleomicina, etoposido y cisplatino, sin observar recurrencia. No existen en la actualidad estudios prospectivos aleatorios que comparen la aplicación de tratamiento adyuvante versus seguimiento controlado, para el manejo de pacientes en estadío clínico I sometidos a LRP-L, con hallazgos positivos en el examen anatomopatológico. Es en este punto donde se plantea el mayor cuestionamiento en cuanto a la eficacia oncológica de la $\operatorname{LRP}-L(2,12)$. La eficacia demostrada por la quimioterapia adyuvante para los pacientes en estadío II posterior a la LRP abierta, hacen cuestionable el tratamiento quirúrgico en un grupo de pacientes en los que se ha demostrado mas de $95 \%$ de posibilidades de curación (18).

La eficacia oncológica de la LRP-L, como lo plantean Janetschek y cols. (20) puede evaluarse al comprobar la tasa de recurrencia en el grupo de pacientes que conservan el estadío luego de la cirugía. En nuestra serie, 4 pacientes $(3.6 \%)$ presentaron recurrencia retroperitoneal, situación atribuible al método quirúrgico. La tasa de recurrencia luego de la LRP abierta no supera el $10 \%$ en diferentes series para los pacientes en estadío anatomopatológico I (11).

Los análisis de costos que comparan la técnica laparoscópica con la abierta para la realización de la LRP, si bien son diferentes desde el punto de vista metodológico, han arrojado diferencias mínimas entre ambos procedimientos $(3,4)$. Las puntuales series publicadas sobre LRP-L evidencian resultados comparables en el presente reporte, Tabla III $(3,12-23)$. La mayor diferencia la hemos observado al evaluar nuestro tiempo operatorio, el cual es significativamente menor al publicado en otros centros con experiencia en LRP-L. Todos los reportes son coincidentes con relación a la complejidad del procedimiento y a la necesidad de una curva de aprendizaje prolongada, situación que obliga al manejo de este grupo de pacientes en centros con amplia experiencia en cirugía laparoscópica.

\section{CONCLUSIÓN}

La LRP-L constituye una adecuada alternativa para el manejo de los pacientes con TGNS en estadío I permitiendo lograr una adecuada estadificación con una morbilidad inferior a la de la cirugía tradicional. Desde el punto de vista oncológico, la cirugía laparoscópica ofrece resultados equivalentes a la cirugía abierta en la disección del tejido ganglionar linfático retroperitoneal indicada por tumor de testículo.

\section{BIBLIOGRAFIA y LECTURAS

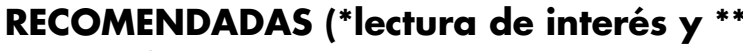 lectura fundamental)}

**1. JANETSCHEK, G.: "Laparoscopic retroperitoneal lymph node dissection". Urol. Clin. North Am., 28: 107, 2001.

**2. FOSTER, R. y BIHRLE, R.: "Current status of retroperitoneal lymph node dissection and testicular cancer: when to operate". Cancer Control, 9: 277, 2002.

*3. JANETSCHEK, G.; HOBISCH, A.; HOLTL, L. y cols.: "Laparoscopic retroperitoneal lymphadenectomy for clinical stage I nonseminomatous testicular tumor: laparoscopy versus open surgery and impact of learning curve. J Urol, 156: 89, 1996.

4. OGAN, K.; LOTAN, Y.; KOENEMAN, K. y cols.: "Laparoscopic versus open retroperitoneal lymph node dissection: A cost analysis". J. Urol., 168: 1945, 2002.

5. CASTILLO, O.; UREÑA, R.; PINTO, I. Y cols.: "Laparoscopic retroperitoneal lymph node dissection for stage I and II NSGCT: 10 years experience". J. Urol. 171: Abstract 933, page 247, 2004.

6. CASTILlO, O.; PINTO, I.; PORTALIER, P. y cols.: "Anterior extraperitoneal laparoscopic retroperitoneal lymph node dissection in testicular cancer: Initial experience in five consecutive patients". J. Endourology 17 (Suppl. 1): Abstract V11.06, page A308, 2003.

**7. WEISSBACH, L. y BOEDEFELD, E.A.: "Localization of solitary and multiple metastases in stage II nonseminomatous testis tumor as basis for a modified staging lymph node dissection in stage I". J. Urol., 138: 77, 1987.

*8. HÖLTL, L.; PESCHEL, R.; KNAPP, R. y cols.: "Primary lymphatic metastatic spread in testicular cancer occurs ventral to the lumbar vessels". Urology, 59: 114, 2002.

9. STUDER, U.E.; BURKHARD, F.C.; y SONNTAG, R.W.: "Risk adapted management with adjuvant chemotherapy in patients with high risk cli- 
nical stage I nonseminomatous germ cell tumor". J. Urol., 163: 1785, 2000.

*10. BÖHLEM, D.; BORNER, M.; SONNTAG, R.W. y cols.: "Long-term results following adjuvant chemotherapy in patients with clinical stage I testicular nonseminomatous malignant germ cell tumor with high risk factors". J. Urol., 161: 1148, 1999.

11. AMATO, R.J.; RO, J.Y.; AYALA, A.G. y cols.: "Risk adapted treatment for patients with $\mathrm{cli}$ nical stage I nonseminomatous germ cell tumor of the testis". Urology 63: 144, 2004.

12. FOSTER, R.S. y DONOHUE, J.P.: "Retroperitoneal lymph node dissection for the management of clinical stage I nonseminoma". J. Urol., 163: $1788,2000$.

**13. HEIDENREICH, A.; ALBERS, P.; HARTMANN, M. y cols.: "Complications of primary nerve sparing retroperitoneal lymph node dissection for clinical stage I nonseminomatous germ cell tumors of the testis: Experience of the German testicular cancer study group". J. Urol., 169: 1710, 2003.

**14. DONOHUE, J.P.; FOSTER, R.S.; ROWLAND, R.G. y cols.: "Nerve-sparing retroperitoneal lymphadenectomy with preservation of ejaculation". J. Urol., 144: 287, 1990.

15. JEWETT, M.A.; KONG, Y.S.; GOLDBERG, S.D.; y cols.: "Retroperitoneal lymphadenectomy for testis tumor with nerve sparing for ejaculation". J. Urol., 139: 1220, 1987.

*16. PESCHEL, R.; GETTMAN, M.T.; NEURURER, R. y cols.: "Laparoscopic retroperitoneal lymph node dissection: description of the nerve sparing technique". Urology 60: 339, 2002.
17. STEINER, H.; PESCHEL, R.; JANETSCHEK, G. y cols.: "Long- term results of laparoscopic retroperitoneal lymph node dissection: A single center 10-year experience". Urology 63: 550, 2004.

18. BHAYANI, S.B.; ONG, A.; OH, W.K. y cols.: "Laparoscopic retroperitoneal lymph node dissection for clinical stage I nonseminomatous germ cell testicular cancer: a long-term update". Urology, 62: 324, 2003.

19. LEBLANC, E.; DARGENT, C.D.; QUERLEU, D. y cols.: "Extraperitoneal laparoscopic para-aortic lymph node dissection for early stage nonseminomatous germ cell tumors of the testis with introduction of a nerve sparing technique: descriptions and results". J. Urol., 165: 89, 2001.

**20. JANETSCHECK, G. y ALBQAMI, N.: "Laparoscopic retroperitoneal lymph-node dissection in the management of clinical stage I and II testicular cancer". J. Endourol. 19:683, 2005.

21. JANETSCHEK, G.; PESCHEL, R.; HOBISCH, A. y cols.: "Laparoscopic retroperitoneal lymph node dissection". J. Endourol., 15: 449, 2001.

22. NELSON, J.B.; CHEN, R.N.; BISHOFF, J.T. y cols.: "Laparoscopic retroperitoneal lymph node dissection for clinical stage I nonseminomatous germ cell tumors". Urology, 54: 1064, 1999.

23. RASSWEILER, J.J.; FREDE, T.; LENZ, E. y cols.: "Long-term experience with laparoscopic retroperitoneal lymph node dissection in the management of low-stage testis cancer". Eur. Urol. 37: 251, 2000. 